\title{
The Influence of Some Foliar Fertilizers on Tomato Yield and Fruit Quality
}

\author{
Gabriela SOVAREL ${ }^{1 *}$, M. COSTACHE ${ }^{1}$, Mihaela CROITORU ${ }^{2}$ \\ ${ }^{1}$ Plant protection Laboratory, Research \& Development Institute for Vegetable and Flower Growing \\ Vidra, Romania \\ ${ }^{2}$ Horticulture Laboratory, Research \& Development Center for Agricultural Plants on Sandy Soils \\ Dabuleni, Romania \\ *)Corresponding author, e-mail: gabriela_sovarel@yahoo.com
}

BulletinUASVM Horticulture 73(1) / 2016

Print ISSN 1843-5254, Electronic ISSN 1843-5394

DOI:10.15835/buasvmcn-hort:11495

\begin{abstract}
The foliar fertilizers applied on tomatoes plant grown under high plastic tunnels improve the quantity and quality of yield. The foliar products with many active substances (N, P, K, Fe, Mg, Zn. Cu, Mn, B, Ca, Mo, Co, Ni, organic aminoacids, vitamins, vegetable enzymes and growth stimulators) application on tomato determined higher yields. Other foliar products with organic matter, natural plant hormones from algae extracts, essentials aminoacids and biostimulators application improve the content in $\mathrm{C}$ vitamin of fruits.
\end{abstract}

Keywords: tomato, fertilizers, high plastic tunnels

\section{INTRODUCTION}

Using foliar fertilizers on vegetable protected crops helps to improve the nutritional status of plants, providing an appropriate nutrition in relation withtherequirements of plantphenophases (vegetative growth, flowering, fruiting). Many new formulations of foliar fertilizers have been recommended and implemented in the practical agriculture and horticulture. This development encouraged the progressive production of foliar fertilizers in many countries (El-Fouly, 2002). The efficiency with which foliar applied nutrients are utilized depends on the mobility of the specific nutrient throughout the entire plant, mobility comprising long distance transport especially phloem transport as well as the symplastic transport (Mengel, 2002). Foliar fertilization is a complementary measure, unconventional and ecological who improve the mineral nutrition and makes the plants to withstand stress conditions and also to ensure satisfactory yields (Anton et al., 2011).

\section{AIMS AND OBJECTIVES}

The research aimed to study the influence of some foliar fertilizers on the tomato yield and quality of fruits.

\section{MATERIAL AND METHODS}

The studied biological material was represented by tomato cultivar Siriana F1. Planting date was April 16, 2014 and planting density $33500 \mathrm{pl} \mathrm{ha}^{-1}$. The variants foliar fertilized with the following substance active:

V1. (2000mg N, 4000mg P, 200mg K, 220mg Fe, $550 \mathrm{mg} \mathrm{Mg}$, 49mg Zn, 35mg Cu, 54mg Mn, $10 \mathrm{mg}$ $\mathrm{B}, \mathrm{Ca}, \mathrm{Mo}, \mathrm{Co}, \mathrm{Ni}$, organic aminoacids, vitamins, vegetable enzymes, growth stimulators) $0.2 \%$;

V2. (12N:52P:0,5K: Me - 1 application, 20:20:20: Me - 1 application, 11:05:19:9CaO: Me - 2 applications, 15:10:31: Me - 2 applications) $0.3-0.5 \%$;

V3. (30\% P, $20 \% \mathrm{~K}) 0.2 \%$,

V4. (38\% organic matter, 3,2\% N, 1,6\% P, 3,2\% K, 6,5\% aminoacids, $150 \mathrm{mg} / \mathrm{l} \mathrm{Fe}, 1200 \mathrm{mg} / \mathrm{l} \mathrm{Mg}$, $25 \mathrm{mg} / \mathrm{l} \mathrm{Zn}, 4,3 \mathrm{mg} / \mathrm{l} \mathrm{Cu}, 33 \mathrm{mg} / \mathrm{l} \mathrm{Mn}, 71 \mathrm{mg} / \mathrm{l} \mathrm{B}$, $670 \mathrm{mg} / \mathrm{l} \mathrm{Ca}, 16 \mathrm{mg} / \mathrm{l} \mathrm{Mo)} \mathrm{0.25 \% ;}$

V5. (natural plant hormones from algae extracts Ascophylum nodosum) 0.4\%;

V6. (42 g/l N, 100 g/l P, 70 g/l K, 5 g/l Mg, Zn, $\mathrm{Fe}, \mathrm{Cu}, \mathrm{Mn}, \mathrm{B}, \mathrm{Mo}, 6$ essentials aminoacids and biostimulators) $0.3 \%$;

V7. (9\% N, 6\% K, 1\% Ag) 0.045\%;

V8. unfertilised check. 
For each foliar product, there were made 6 applications in the following stages: 1. First inflorescence, first flower open (BBCH 61); 2. Third inflorescence, first flower open $(\mathrm{BBCH}$ 63); 3. First fruit cluster, first fruit has typical size (BBCH 71); 4. Second fruit cluster, first fruit has typical size (BBCH 72); 5. 10\% of fruits show typical fully ripe color (BBCH 81); $6.20 \%$ of fruits show typical fully ripe color (BBCH 82 ). The plants top were cut - off after 6 inflorescences.

\section{RESULTS AND DISCUSSION}

The highest number of fruits/plant were obtained at V1 (28.3), V2 (27.0) and V6 (27.0). The highest average weight of fruits presented the variants V6 (150.3g) and V2 (149.2g). The yield varied between $11.75 \mathrm{~kg} \cdot \mathrm{m}^{-2}$ (without foliar fertilization) and $13.8 \mathrm{~kg} \cdot \mathrm{m}^{-2}(\mathrm{~V} 1)$.
The content in $\mathrm{C}$ vitamin varied between $16.72 \mathrm{mg} / 100 \mathrm{~g}$ fresh material (without foliar fertilization) and $32.56 \mathrm{mg} / 100 \mathrm{~g}$ fresh vegetable material (V3).

\section{CONCLUSION}

The foliar fertilizers $(42 \mathrm{~g} / \mathrm{l} \mathrm{N}, 100 \mathrm{~g} / \mathrm{l} \mathrm{P}, 70$ $\mathrm{g} / \mathrm{l} \mathrm{K,} 5 \mathrm{~g} / \mathrm{l} \mathrm{Mg}, \mathrm{Zn}, \mathrm{Fe}, \mathrm{Cu}, \mathrm{Mn}, \mathrm{B}, \mathrm{Mo}, 6$ essentials aminoacids and biostimulators) $0.3 \%,(2000 \mathrm{mg} \mathrm{N}$, $4000 \mathrm{mg}$ P, 200mg K, 220mg Fe, 550mg Mg, 49mg $\mathrm{Zn}, 35 \mathrm{mg} \mathrm{Cu}, 54 \mathrm{mg} \mathrm{Mn}, 10 \mathrm{mg} \mathrm{B}, \mathrm{Ca}, \mathrm{Mo}, \mathrm{Co}, \mathrm{Ni}$, organic aminoacids, vitamins, vegetable enzymes, growth stimulators) $0.2 \%$ and $(12 \mathrm{~N}: 52 \mathrm{P}: 0,5 \mathrm{~K}: \mathrm{Me}$ - 1 application, 20:20:20:Me - 1 application, 11:05:19:9CaO:Me - 2 applications,15:10:31:Me - 2 applications) $0.3-0.5 \%$ applied on tomato crop grown under high plastic tunnels improve the yield $\left(13.8 \mathrm{~kg} \cdot \mathrm{m}^{-2}, 13.59 \mathrm{~kg} \cdot \mathrm{m}^{-2}\right.$ and 13.49 $\mathrm{kg} \cdot \mathrm{m}^{-2}$ ). The foliar products with following active

Tab. 1. The influence of foliar fertilizers on tomato yield

\begin{tabular}{|c|c|c|c|c|c|c|}
\hline $\begin{array}{l}\text { No. } \\
\text { crt. }\end{array}$ & Foliar fertilizer & $\begin{array}{c}\text { No. fruits/ } \\
\text { plant }\end{array}$ & $\begin{array}{l}\text { Nr. fruits/ } \\
\text { cluster }\end{array}$ & $\begin{array}{c}\text { Average fruits } \\
\text { weight }(\mathrm{g})\end{array}$ & $\begin{array}{c}\text { Yield/plant } \\
(\mathrm{kg})\end{array}$ & $\begin{array}{c}\text { Yield/sqm } \\
(\mathrm{kg})\end{array}$ \\
\hline 1. & V1 & 28.2 & 4.7 & 146.1 & 4.12 & $13.8^{\mathrm{xx}}$ \\
\hline 2. & $\mathrm{~V} 2$ & 27.0 & 4.5 & 149.2 & 4.03 & $13.5^{\mathrm{x}}$ \\
\hline 3. & V3 & 25.8 & 4.3 & 143.6 & 3.70 & 12.4 \\
\hline 4. & V4 & 26.4 & 4.4 & 145.6 & 3.84 & 12.8 \\
\hline 5. & V5 & 25.2 & 4.2 & 145.7 & 3.67 & 12.3 \\
\hline 6. & V6 & 27.0 & 4.5 & 150.3 & 4.06 & $13.6^{x}$ \\
\hline 7. & V7 & 25.8 & 4.3 & 147.7 & 3.81 & 12.7 \\
\hline 8. & Check unfertilized & 24.6 & 4.1 & 142.6 & 3.51 & 11.7 \\
\hline & $\begin{array}{l}\text { LSD 5\% } \\
\text { LSD 1\% } \\
\text { LSD 0.1\% }\end{array}$ & & & & & $\begin{array}{l}1.80 \\
2.03 \\
2.44\end{array}$ \\
\hline
\end{tabular}

Tab. 2. The influence of foliar fertilizers on the biochemical composition of tomato fruits

\begin{tabular}{ccccccccc}
\hline Foliar fertilizer & $\begin{array}{c}\text { Water } \\
(\%)\end{array}$ & $\begin{array}{c}\text { Total dry } \\
\text { matter } \\
(\%)\end{array}$ & $\begin{array}{c}\text { Soluble } \\
\text { matter } \\
(\%)\end{array}$ & $\begin{array}{c}\text { Acidity } \\
\text { (g citric acid/100g } \\
\text { fresh matter) }\end{array}$ & $\mathrm{pH}$ & $\begin{array}{c}\text { Glucids } \\
(\%)\end{array}$ & $\begin{array}{c}\text { Nitrats } \\
\text { (mg/kg) }\end{array}$ & $\begin{array}{c}\text { C Vitamin } \\
\text { (mg/100g } \\
\text { fresh matter) }\end{array}$ \\
\hline V1 & 94.51 & 5.49 & 4.1 & 0.34 & 3.84 & 3.4 & 29.00 & 22.00 \\
\hline V2 & 94.01 & 5.99 & 3.7 & 0.53 & 3.93 & 3.07 & 34.00 & 19.36 \\
\hline V3 & 94.84 & 5.16 & 4.0 & 0.64 & 3.91 & 3.32 & 33.00 & 32.56 \\
\hline V4 & 95.46 & 4.54 & 3.2 & 0.34 & 3.84 & 2.65 & 36.00 & 30.80 \\
\hline V5 & 95.03 & 4.97 & 3.1 & 0.51 & 3.93 & 2.57 & 36.38 & 29.04 \\
\hline V6 & 94.58 & 5.42 & 3.2 & 0.53 & 3.90 & 2.65 & 34.00 & 29.04 \\
\hline V7 & 94.47 & 5.53 & 3.6 & 0.64 & 3.82 & 2.98 & 36.00 & 18.48 \\
\hline Check unfertilized & 95.03 & 4.97 & 3.0 & 0.43 & 3.92 & 2.49 & 34.50 & 16.72 \\
\hline
\end{tabular}


substances $(30 \% \mathrm{P}, 20 \% \mathrm{~K}) \quad 0.2 \%,(38 \%$ organic matter, 3,2\% N, 1,6\% P, 3,2\% K, 6,5\% aminoacids, $150 \mathrm{mg} / \mathrm{l} \mathrm{Fe}, 1200 \mathrm{mg} / \mathrm{l} \mathrm{Mg}, 25 \mathrm{mg} / \mathrm{l} \mathrm{Zn}, 4,3 \mathrm{mg} / \mathrm{l}$ $\mathrm{Cu}, 33 \mathrm{mg} / \mathrm{l} \mathrm{Mn}, 71 \mathrm{mg} / \mathrm{l} \mathrm{B}, 670 \mathrm{mg} / \mathrm{l} \mathrm{Ca}, 16 \mathrm{mg} / \mathrm{l}$ Mo) $0.25 \%$, (natural plant hormones from algae extracts Ascophylum nodosum) $0.4 \%$ and (42 g/l N, 100 g/l P, 70 g/l K, 5 g/l Mg, Zn, Fe, Cu, Mn, B, Mo, 6 essentials aminoacids and biostimulators) $0.3 \%$ improves the content in $\mathrm{C}$ vitamin of fruits $(32.56$ $\mathrm{mg}, 30.80 \mathrm{mg}$ and $29.04 \mathrm{mg} / 100 \mathrm{~g}$ fresh matter).

\section{REFERENCES}

1. Anton Iulia, Dorneanu A, Bireescu Geanina, Sirbu Carmen, Stroe Venera, Grigore Adriana (2011).

2. Foliar fertilization effect on production and metabolism on tomatoes plants. Research Journal of Agricultural Science 43(3), 3-10

3. 2. El-fouly MM. (2002). Quality of foliar fertilizers. Acta hortic. 277-281.

4. 3. Mengel K (2002). Alternative or complementary role of foliar supply in mineral nutrition. Acta hortic. 365 -373. 